\title{
MULTIPACTOR SIMULATIONS IN SUPERCONDUCTING CAVITIES
}

\author{
I.Gonin", N.Solyak - FNAL, Batavia, IL 60510, U.S.A. \\ J. DeFord, B. Held - STAAR Inc., Mequon, WI 53092, U.S.A.
}

\begin{abstract}
The multipactor (MP) is a well-known phenomenon. The existence of resonant trajectories can lead to electron avalanche under certain field levels and surface conditions, and can limit the performance of high power superconducting (SC) radio-frequency (RF) devices. In this paper we describe features of the ANALYST particle tracking code PT3P developed for MP simulations in real 3D RF structures, such as cavities, couplers, RF windows etc. Also we present the results of MP simulations in HOM couplers of TESLA, SNS and FNAL $3^{\text {rd }}$ harmonic cavities. We discuss the comparison of simulations with experimental results.
\end{abstract}

\section{INTRODUCTION}

Recently the interest in evaluation of MP phenomena in RF structures has significantly increased. The high current of modern linacs requires more power, resulting in higher field levels inside of the cavity and related complex 3D structures like HOM couplers and RF windows, increasing the chance of MP.

The existing analysis capabilities for studying MP in complicated 3D RF structures are noticeably improved. The ANALYST [1] parallel finite-element particle tracking code PT3P has been developed and a variety of tests have been performed showing a good agreement with both analytical calculations and experimental data. A combination of three recent technologies that have been developed in the ANALYST code are used: high-order adaptive finite-element RF field calculations, advanced particle tracking on unstructured grids, and secondary emission models. ANALYST employs high-order adaptive finite-element methods to accurately compute RF fields in complex geometries, particularly near edges, corners, and curved surfaces. To perform a MP analysis, we use the mesh and fields from the ANALYST eigensolver OM3P[2,3] in PT3P, which has both a fast, accurate, and reliable particle tracker for unstructured grids and a secondary emission model.

\section{NUMERICAL APPROACH}

The approach to efficiently and accurately track particles on finite-element (FE) meshes relies on the identification of exact element entrance and exit points. Starting with the location and initial conditions of the particle as it enters an element; the path is computed by integrating the relativistic velocity and acceleration equations within the element:

\# gonin@fnal.gov

$$
\dot{\vec{r}}=\frac{\vec{p}}{\gamma m} \quad \ddot{\vec{r}}=\frac{q}{\gamma m}\left[\vec{E}+\frac{1}{\gamma m} \vec{p} \times \vec{B}-\frac{\vec{E} \circ \vec{p}}{m^{2} c^{2}\left(1+\gamma^{2} \beta^{2}\right)} \vec{p}\right]
$$

with $\vec{p}$ being the particle momentum.

The integration is performed by first assuming a quadratic variation in the particle path between element entrance and exit points. The time, location, and momentum are determined at the exit point, and these are used to determine a $3^{\text {rd }}$ order path correction. If the correction is too large, then an internal point is introduced in the element and the procedure is repeated between the new path end-points.

The electric and magnetic fields are evaluated directly from the associated finite-element vector basis functions within each element, so no interpolation of the fields is required.

We assume that the secondary electron yield (SEY) can be characterized by a single curve that is only dependent on impact energy and has a simple parameterization:

$$
S E Y=Y_{p}(2.72)^{2} \frac{K}{K_{\max }} e^{-2 \sqrt{K / K_{\max }}}
$$

where $Y_{p}$ is the maximum yield value, $K_{\max }$ is the impact kinetic energy that gives the maximum yield, and $K$ is the kinetic energy of the impacting electron.

Initial emission of particles from all MP surfaces in a model is done over a single RF period. Once a particle is emitted, it is tracked until termination based on several criteria, including the RF phase on impact, the impact location, the total number of impacts, the cumulative yield, and optionally the RF phase advance between impacts. If not terminated, a single particle is emitted at the impact site and tracking continues, with each emitted particle linked to its parent impacting particle in a chain. Resonant particles are defined as those that survive a minimum number of impacts/emissions as specified by the user. The yield is also tallied at each impact in a particle chain.

The software outputs the following set of MP statistics:

- The counter function (CF), defined as the number of particles that survive at least $n$ impacts at a particular field level.

- The enhanced counter function (ECF), which is similar to the counter function except that it includes the effect of secondary yield at each impact.

- A yield function (YF), which is equal to the maximum average yield per impact for each resonant particle. 
The functions are reported vs. field level, showing potential MP bands as spikes in the plots at problematic field levels. The elevated values of EF or YF indicate potential MP bands that typically warrant further investigation.

\section{MP IN TESLA CAVITY}

Fig. 1 shows the solid model used for ANALYST analysis of MP in the HOM coupler of $1.3 \mathrm{GHz}$ TESLA cavities. Because the cavities operate in $\pi$-mode, only one cell has to be added to the end group for calculation of RF fields in the coupler.

ANALYST simulations predict MP activity at $\mathrm{E}_{\mathrm{acc}} \sim 1.5$ $\mathrm{MV} / \mathrm{m}$ and $\mathrm{E}_{\mathrm{acc}} \sim 33.8 \mathrm{MV} / \mathrm{m}$. Fig. 1 shows the FE mesh of the solid model and regions where MP takes place. The first one occurs in the notch gap (a) and has an impact energy around $200 \mathrm{eV}$. The second takes place in the gap between the antenna and coupler body (b). The impact energy is in a range $290-310 \mathrm{eV}$.

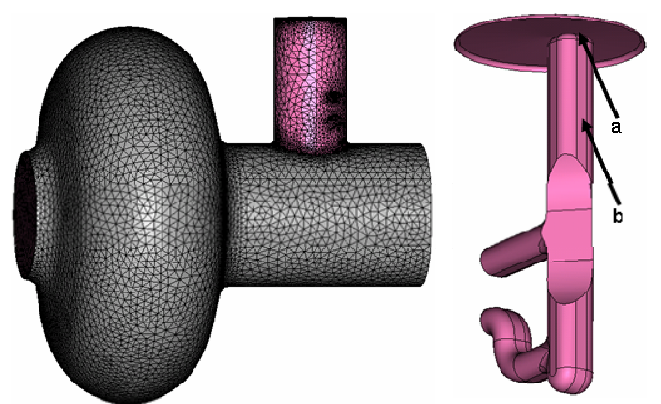

Fig. 1 FE mesh of the TESLA solid model (left) and HOM antenna view showing MP locations a and b (right)

The electric field distribution in both gaps is shown in Fig. 2. In the $1.73 \mathrm{~mm}$ notch gap, the field strength is almost uniform and equals $8 \%$ of the maximum surface field in the structure. In the $8.2 \mathrm{~mm}$ gap between the antenna and coupler body, the field strength varies in the range of $1.2-2.1 \%$ of the maximum surface structure field.

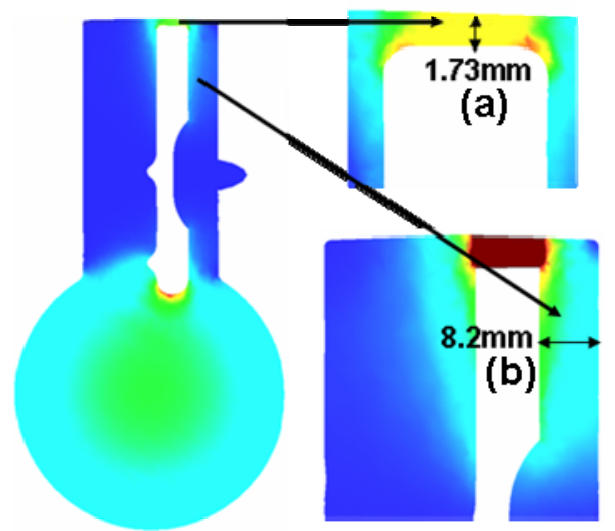

Fig. 2 E-fields in the plane of the TESLA antenna HOM coupler with zooms of the $1.73 \mathrm{~mm}$ and $8.2 \mathrm{~mm}$ gaps

Due to the field uniformity, the MP in the $1.73 \mathrm{~mm}$ gap can be estimated analytically as the MP between two parallel plates. Taking into account the secondary emission yield, analytical calculations show that the first order MP in the $1.73 \mathrm{~mm}$ gap can be expected at a field level $\mathrm{E}_{\mathrm{acc}} \sim 1.1-1.7 \mathrm{MV} / \mathrm{m}$.

The simulated and analytical results agree well with the test data. Fig. 3 shows the result of one of the vertical tests of cavity Z94 at DESY and a plot of the Enhanced Counter Function (ECF) calculated by ANALYST. Fig. 4 shows similar charts for the cavity A7 tested at Jefferson Lab [4].

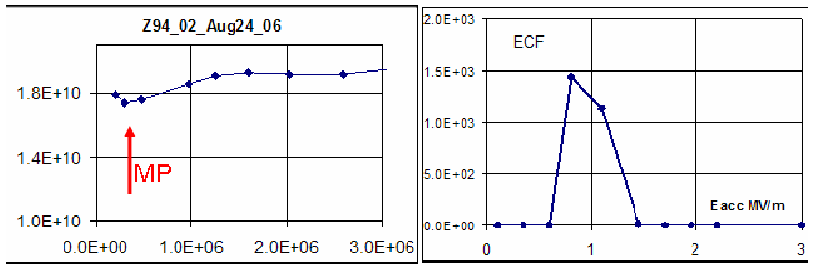

Fig. 3 Q vs. $E_{\text {acc }}$ test results of cavity Z94 (left) and the ECF plot calculated by ANALYST (right). Q-drop is marked by arrow.

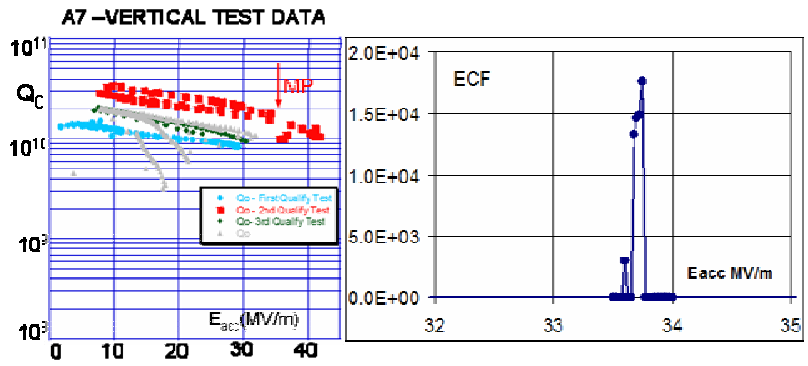

Fig. 4 Q vs. $E_{\text {acc }}$ test results of cavity A7 (left), and the ECF plot calculated by ANALYST (right). Q-drop is marked by arrow.

The Q-drops around $\mathrm{E}_{\mathrm{acc}} \sim 0.5 \mathrm{MV} / \mathrm{m}$ for cavity Z94 and $\mathrm{E}_{\mathrm{acc}} \sim 34 \mathrm{MV} / \mathrm{m}$ for cavity A7 are very well agreed with prediction made by simulations.

\section{MP IN THIRD HARMONIC CAVITY}

The $3^{\text {rd }}$ harmonic $3.9 \mathrm{GHz}$ cavity is under production at Fermilab. During the vertical tests of cavities, MP activity was observed at $\mathrm{E}_{\mathrm{acc}} \sim 0.7 \mathrm{MV} / \mathrm{m}$ and at $\mathrm{E}_{\mathrm{acc}} \sim 12-13 \mathrm{MV} / \mathrm{m}$. The results of test measurements for cavity \#3 are shown in Fig. 5. The first MP level causes a Q-drop and the second MP results in a quench.

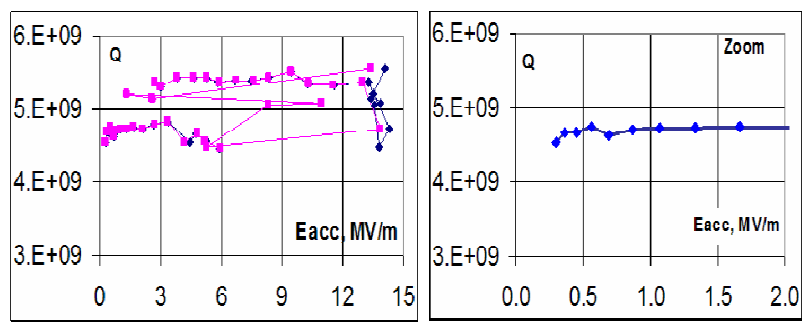

Fig.5: Q vs. $\mathrm{E}_{\mathrm{acc}}$ measurements for cavity \#3 (left), and a zoom of the range $E_{\text {acc }}=0-2 \mathrm{MV} / \mathrm{m}$ (right)

ANALYST simulations of MP in the HOM coupler show MP at 2 field levels very close to test values. The 
first MP occurs in the $0.6 \mathrm{~mm}$ notch gap at $\mathrm{E}_{\text {acc }} \sim 0.7$ $\mathrm{MV} / \mathrm{m}$. The second one is wider, occurring in the range $\mathrm{E}_{\text {acc }} \sim 11-18 \mathrm{MV} / \mathrm{m}$. This is a two-point MP taking place in the $2 \mathrm{~mm}$ gap between antenna leg and coupler body. The resonant trajectories and ECF are shown in Fig.6.

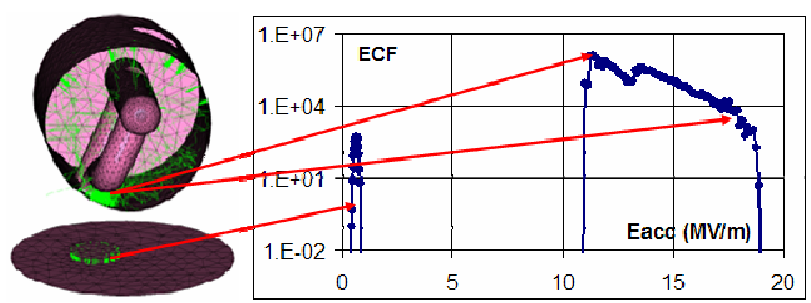

Fig. 6 Resonant trajectories in the range $\mathrm{E}_{\mathrm{acc}}=11-$

$18 \mathrm{MV} / \mathrm{m}$ (top left), at $\mathrm{E}_{\mathrm{acc}}=0.7 \mathrm{MV} / \mathrm{m}$ in the notch gap (bottom left), and ECF after 40 impacts (right), for the $3^{\text {rd }}$ harmonic cavity.

Presentation WEPMN111 at this conference describes in more detail the design of a new HOM coupler to overcome MP problems.

\section{MP IN SNS CAVITIES}

SC medium-beta (0.61) and high-beta (0.81) $805 \mathrm{MHz}$ cavities for SNS have been produced at Jefferson Lab. Analysis of RF test data [5] for high-beta cavities show that almost all of them exhibited MP during testing and that the onset occurred from 9.5 to $18.6 \mathrm{MV} / \mathrm{m}$ with a mean of $11.7 \mathrm{MV} / \mathrm{m}$ and a standard deviation of 1.8 $\mathrm{MV} / \mathrm{m}$. SNS cavities consist of 6 cells and have end tubes of different diameters.

ANALYST simulations were made for both structures up to $E_{\text {acc }}=30 \mathrm{MV} / \mathrm{m}$ which show a MP location in HOM couplers at the end tubes with bigger diameter.

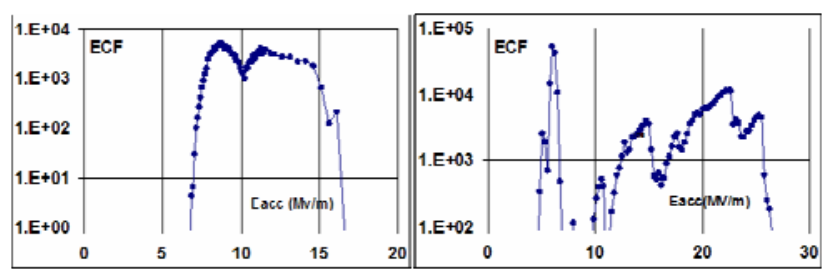

Fig. 7 ECF for medium-beta (left) and high-beta (right)

For medium beta MP takes place in the $4 \mathrm{~mm}$ gap between antenna leg and coupler body and has a wide range (see Fig. 7). The electric field strength in the gap has a range $\mathrm{E}_{\mathrm{gap}} / \mathrm{E}_{\mathrm{acc}} \sim 0.01-0.02$. For high beta, ANALYST finds two locations of MP. The first one occurs in the same place, and has a wide range of field strength $\mathrm{E}_{\mathrm{gap}} / \mathrm{E}_{\mathrm{acc}} \sim 0.006-0.016$. The second one is narrow and takes place in the $5.3 \mathrm{~mm}$ gap with a field strength of $\mathrm{E}_{\mathrm{gap}} / \mathrm{E}_{\mathrm{acc}} \sim 0.041$. In simulations we didn't observe MP in the $5.3 \mathrm{~mm}$ gap for the medium-beta cavity, which can be explained by the 3 times lower field strength. Electric fields in the 4 and $5.3 \mathrm{~mm}$ gaps for the high-beta cavity are shown in Fig. 8. Fig. 9 demonstrates resonant trajectories at $\mathrm{E}_{\text {acc }}=12 \mathrm{MV} / \mathrm{m}$ for medium-beta and at $E_{\text {acc }}=6 \mathrm{MV} / \mathrm{m}$ and $E_{\text {acc }}=15 \mathrm{MV} / \mathrm{m}$ for high-beta cavities.

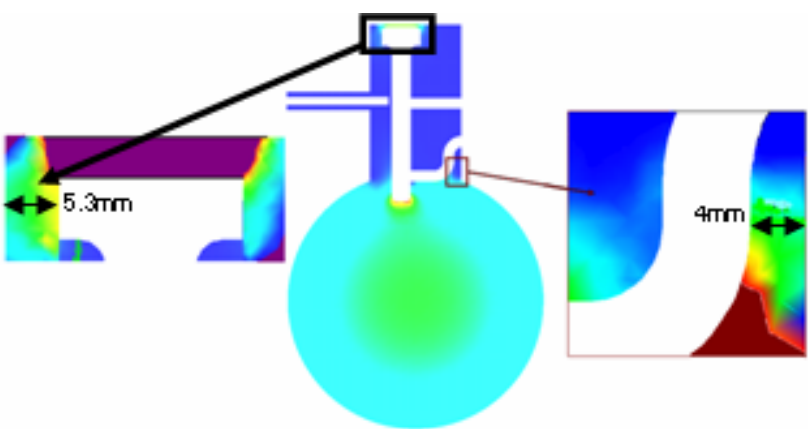

Fig. 8 Electric field distribution in the high-beta HOM coupler in the antenna plane (center) and in zooms of the gaps where MP takes place (left and right)

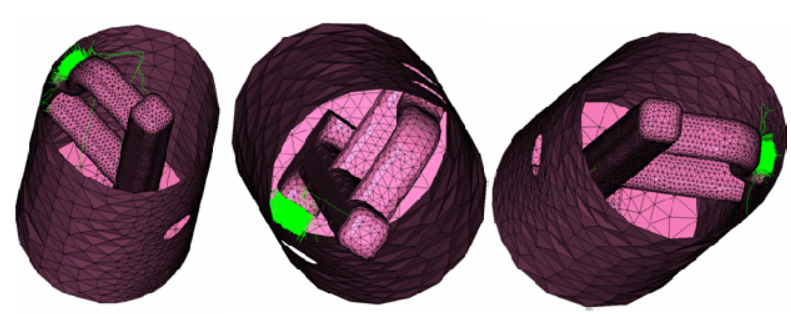

Fig. 9 Resonant trajectories for medium-beta cavities at $\mathrm{E}_{\mathrm{acc}}=12 \mathrm{MV} / \mathrm{m}$ (left), and for high-beta cavities at $\mathrm{E}_{\mathrm{acc}}=6 \mathrm{MV} / \mathrm{m}$ (middle) and $\mathrm{E}_{\mathrm{acc}}=15 \mathrm{MV} / \mathrm{m}$ (right)

Again, the test results for SNS cavities are well correlated with simulations and analytical predictions of MP in the case of two parallel plates with gaps of 4 and $5.3 \mathrm{~mm}$ with the assumption of uniform fields.

\section{CONCLUSION}

In recent years a very powerful tool for the evaluation of MP phenomena has been developed. Having advantages of high-order adaptive $\mathrm{FE}$ methods to accurately compute RF fields and advanced particle tracking, ANALYST allows simulating the MP behavior in complex 3D RF structures with high accuracy. ANALYST simulations of MP in HOM couplers of TESLA, SNS and FNAL $3^{\text {rd }}$ harmonic cavities has been done and show the MP activity for all of them. In all cases the results of simulations are in good agreement with available test measurement data.

The new tool provides new capabilities to better predict the MP behavior in complex 3D structures, allowing new cavities to be more reliably designed.

\section{REFERENCES}

[1] http://www.staarinc.com/

[2] J.DeFord et al., Proc. of PAC 2003, Portland, OR, pp. 3554-3556.

[3] J. DeFord et al., Proc. of ICAP 2002, East Lansing, MI, pp. 57-62.

[4] H.Padamsee, Regional Report US, TTC Meeting at FNAL, April 23-26, 2007.

[5] J.R.Delayen et al., MOP81, Linac 2004, Lubeck, Germany. 